International Mathematical Forum, 2, 2007, no. 36, 1773 - 1777

\title{
On Galois Extensions Satisfying the Fundamental Theorem
}

\author{
George Szeto \\ Department of Mathematics \\ Bradley University \\ Peoria, Illinois 61625, USA \\ szeto@bradley.edu \\ Lianyong Xue \\ Department of Mathematics \\ Bradley University \\ Peoria, Illinois 61625, USA \\ lxue@bradley.edu
}

\begin{abstract}
Let $B$ be a Galois extension of $B^{G}$ with Galois group $G$. It is shown that $B$ satisfies the fundamental theorem if and only if $B$ is either indecomposable satisfying the fundamental theorem, or $B=B^{G} e \oplus$ $B^{G}(1-e)$ where $e$ and $1-e$ are minimal central idempotents in $B$, and $G$ has order 2 .
\end{abstract}

Mathematics Subject Classification: 16S35, 16W20

Keywords: Galois extensions, Galois algebras, separable extensions

\section{Introduction}

In [1], it was shown that the fundamental theorem holds for any indecomposable commutative Galois extension (with no idempotents but 0 and 1 ). Recently, in [5], a Galois algebra $B$ over a commutative ring $R$ with Galois group $G$ satisfying the fundamental theorem is characterized; that is, $B$ satisfies the fundamental theorem if and only if $B$ is one of the following three types: (1) $B$ is an indecomposable commutative Galois algebra, (2) $B=R e \oplus R(1-e)$ where $e$ and $1-e$ are minimal central idempotents in $B$, and (3) $B$ is indecomposable noncommutative such that $A=\oplus \sum_{g \in G\left(A^{\prime}\right)} J_{g}$ and the centers of $A$ and 
$B^{G(A)}$ are the same for each separable subalgebra $A$, where $A^{\prime}=V_{B}(A)$, the commutator subalgebra of $A$ in $B, G\left(A^{\prime}\right)=\left\{g \in G \mid g(a)=a\right.$ for all $\left.a \in A^{\prime}\right\}$, and $J_{g}=\{b \in B \mid b x=g(x) b$ for each $x \in B\}$ ([5], Theorem 4.7). The purpose of the present paper is to generalize the above characterization to any Galois extension, not necessary a Galois algebra. We shall show that, for any Galois extension $B$ of $B^{G}$ with Galois group $G, B$ satisfies the fundamental theorem if and only if $B$ is one of the following two types: (1) $B$ is indecomposable satisfying the fundamental theorem, or (2) $B=B^{G} e \oplus B^{G}(1-e)$ where $e$ and $1-e$ are minimal central idempotents in $B$, and $G$ has order 2 .

\section{Definitions and Notations}

Let $B$ be a ring with $1, G$ a finite automorphism group of $B, B^{G}$ the set of elements in $B$ fixed under each element in $G$, and $A$ a subring of $B$ with the same identity 1 . We call $B$ a Galois extension of $B^{G}$ with Galois group $G$ if there exist elements $\left\{a_{i}, b_{i}\right.$ in $\left.B, i=1,2, \ldots, m\right\}$ for some integer $m$ such that $\sum_{i=1}^{m} a_{i} g\left(b_{i}\right)=\delta_{1, g}$ for each $g \in G([2])$. A ring $B$ is called a Galois algebra over $R$ if $B$ is a Galois extension of $R$ which is contained the center of $B([3])$. We call $B$ a separable extension of $A$ if there exist $\left\{a_{i}, b_{i}\right.$ in $B, i=1,2, \ldots, m$ for some integer $m\}$ such that $\sum a_{i} b_{i}=1$, and $\sum b a_{i} \otimes b_{i}=\sum a_{i} \otimes b_{i} b$ for all $b$ in $B$ where $\otimes$ is over $A$. A ring $B$ is called indecomposable if it contains no central idempotents but 0 and 1 . We call $B$ satisfying the fundamental theorem if $\alpha: H \longrightarrow B^{H}$ for a subgroup $H$ of $G$ is a one-to-one correspondence between the set of subgroups of $G$ and the set of separable subextensions of $B^{G}$ in $B$.

Throughout this paper, we assume that $B$ is a Galois extension of $B^{G}$ with Galois group $G$.

\section{The Fundamental Theorem}

In this section, keeping all the definitions and notations in section 2 , we shall show some properties of $B$ satisfying the fundamental theorem, leading to the characterization: $B$ satisfies the fundamental theorem if and only if $B$ is one of the following two types: (1) $B$ is indecomposable satisfying the fundamental theorem, or (2) $B=B^{G} e \oplus B^{G}(1-e)$ where $e$ and $1-e$ are minimal central idempotents in $B$, and $G$ has order 2 .

We begin with a lemma that will play an important role.

Lemma 3.1 Let e be a nonzero central idempotent in $B$. If $\left.g\right|_{B e}$ is an identity for a $g \in G$, then $g=$ identity in $G$.

Proof. See Lemma 4.1 in [4]. 
Lemma 3.2 If $B$ satisfies the fundamental theorem, then for any nonzero central idempotent $e$ in $B, B e=B^{G} e$.

Proof. Let $e$ be a nonzero central idempotent in $B$. Then $B^{G} e \oplus B(1-e)$ is a separable extension of $B^{G}$ such that $\left.G\left(B^{G} e \oplus B(1-e)\right)\right|_{B(1-e)}$ is identity. Hence $G\left(B^{G} e \oplus B(1-e)\right)=\{1\}$ by Lemma 3.1. Since $B$ satisfies the fundamental theorem, $B=B^{G} e \oplus B(1-e)$. Thus $B e=B^{G} e$.

Next we investigate the number of central idempotents in $B^{G}$ and $B$ respectively.

Lemma 3.3 If $B$ satisfies the fundamental theorem, then $B^{G}$ is indecomposable.

Proof. Let $e$ be a nontrivial central idempotent in $B^{G}$. Then $e$ and $1-e$ are nonzero central idempotents in $B$. Thus $B e=B^{G} e$ and $B(1-e)=B^{G}(1-e)$ by Lemma 3.2, and so $B=B e \oplus B(1-e)=B^{G} e \oplus B^{G}(1-e)=B^{G}$. This is a contradiction. Therefore $B^{G}$ is indecomposable.

Lemma 3.4 If B satisfies the fundamental theorem, then B has only finitely many minimal central idempotents.

Proof. Let $I$ be the set of minimal central idempotents in $B, e \in I$, and $O_{e}$ the $G$-orbit of $e$; that is, $O_{e}=\{g(e) \mid g \in G\}$. Then $O_{e}$ contains at most $n$ elements where $n$ is the order of $G$ and $\sum_{e^{\prime} \in O_{e}} e^{\prime}$ is an idempotent in $B^{G}$. But $B^{G}$ is indecomposable by Lemma 3.3, so $\sum_{e^{\prime} \in O_{e}} e^{\prime}=1$. This implies that $O_{e}=I$, and so $I$ is a finite set.

Corollary 3.5 If $B$ satisfies the fundamental theorem, then the G-action on the set of minimal central idempotents in $B$ is transitive.

Lemma 3.6 If $B$ satisfies the fundamental theorem, then $B$ has at most two minimal central idempotents.

Proof. By Lemma 3.4, $B$ has finitely many minimal central idempotents. Let $I=\left\{e_{1}, e_{2}, \cdots, e_{m}\right\}$ for some integer $m$ be the set of minimal central idempotents in $B$. Then $B=\oplus \sum_{i=1}^{m} B e_{i}=\oplus \sum_{i=1}^{m} B^{G} e_{i}$ by Lemma 3.2. In case $m=1$; we are done. In case $m>1$; we first show that $B^{G}\left(e_{1}+e_{2}\right)$ is a proper subring of $B^{G} e_{1} \oplus B^{G} e_{2}$. In fact, it is clear that $B^{G}\left(e_{1}+e_{2}\right) \subset$ $B^{G} e_{1} \oplus B^{G} e_{2}$. Assume $e_{1} \in B^{G}\left(e_{1}+e_{2}\right)$. Then $e_{1}=r\left(e_{1}+e_{2}\right)$ for some $r \in B^{G}$. Hence $e_{1}=e_{1}^{2}=e_{1} \cdot r\left(e_{1}+e_{2}\right)=r e_{1}$. Thus $(1-r) e_{1}=0$. By Corollary 3.5, the $G$-action on $I$ is transitive, so for each $e_{i}, i=2,3, \cdots, m$, there exists some $g_{i} \in G$ such that $g_{i}\left(e_{1}\right)=e_{i}$. Thus $(1-r) e_{i}=0$ for each $i=1,2,3, \cdots, m$. Noting that $\left\{e_{1}, e_{2}, \cdots, e_{m}\right\}$ are all the minimal central idempotents in $B$, we 
have that $1-r=0$; and so $r=1$. But then $e_{1}=r\left(e_{1}+e_{2}\right)=e_{1}+e_{2}$. Therefore $e_{2}=0$, a contradiction. This implies that $e_{1} \notin B^{G}\left(e_{1}+e_{2}\right)$; and so $B^{G}\left(e_{1}+e_{2}\right)$ is a proper subring of $B^{G} e_{1} \oplus B^{G} e_{2}$. Next we claim that $m \leq 2$. Assume $m>2$. Then $B=B e_{1} \oplus B e_{2} \oplus B\left(1-e_{1}-e_{2}\right)=B^{G} e_{1} \oplus B^{G} e_{2} \oplus B^{G}\left(1-e_{1}-e_{2}\right)$ by Lemma 3.2 where $1-e_{1}-e_{2} \neq 0$. Considering the proper separable extension $B^{G}\left(e_{1}+e_{2}\right) \oplus B\left(1-e_{1}-e_{2}\right)$ of $B^{G}$ in $B$, we have that $G\left(B^{G}\left(e_{1}+e_{2}\right) \oplus B\left(1-e_{1}-\right.\right.$ $\left.\left.e_{2}\right)\right)\left.\right|_{B\left(1-e_{1}-e_{2}\right)}=\{1\}$; and so $G\left(B^{G}\left(e_{1}+e_{2}\right) \oplus B\left(1-e_{1}-e_{2}\right)\right)=\{1\}$ by Lemma 3.1. But $G(B)=\{1\}$, so $B=B^{G}\left(e_{1}+e_{2}\right) \oplus B\left(1-e_{1}-e_{2}\right)$. Since $B^{G}\left(e_{1}+e_{2}\right)$ is a proper subring of $B^{G} e_{1} \oplus B^{G} e_{2}, B \neq B^{G}\left(e_{1}+e_{2}\right) \oplus B\left(1-e_{1}-e_{2}\right)$, a contradiction. Thus $m \leq 2$. This completes the proof.

Next we show the main theorem.

Theorem 3.7 Let $B$ be a Galois extension of $B^{G}$ with Galois group $G$. Then $B$ satisfies the fundamental theorem if and only if either $B=B^{G} e \oplus$ $B^{G}(1-e)$ where $e$ and $1-e$ are minimal central idempotents in $B$ and $G=$ $\{1, g\}$ such that $g(e)=1-e$, or $B$ is indecomposable satisfying the fundamental theorem.

Proof. $(\Longrightarrow)$ In case $B$ is indecomposable satisfying the fundamental theorem, we are done. In case $B$ is decomposable; then by Lemma 3.6, $m=1$ or 2 . When $m=1, B=B e_{1}=B^{G} e_{1}=B^{G}$, this is impossible. Thus $m=2$; and so $B=B^{G} e_{1} \oplus B^{G} e_{2}$. Then for any $g \neq 1$ in $G, g\left(e_{1}\right)=e_{2}=1-e_{1}$. Therefore $G=\{1, g\}$.

$(\Longleftarrow)$ It suffices to show the case in which $B=B^{G} e \oplus B^{G}(1-e)$ where $e$ and $1-e$ are minimal central idempotents in $B$ and $G=\{1, g\}$ such that $g(e)=1-e$. In fact, there are exactly two trivial separable extensions over $B^{G}$ in $B: B^{G}$ and $B$ corresponding to exactly two trivial subgroups of $G:\{1, g\}$ and $\{1\}$.

ACKNOWLEDGEMENTS. This paper was written under the support of a Caterpillar Fellowship at Bradley University. The authors would like to thank Caterpillar Inc. for the support.

\section{References}

[1] S.U. Chase, D.K. Harrison and A. Rosenberg, Galois Theory and Galois Cohomology of Commutative Rings, Memoirs Amer. Math. Soc., No. 52, 1965 .

[2] F.R. DeMeyer, Some Notes on the General Galois Theory of Rings, Osaka J. Math., 2 (1965), 117 - 127. 
[3] G. Szeto and L. Xue, The structure of Galois algebras, Journal of Algebra, 237(1) (2001), 238 - 246.

[4] G. Szeto and L. Xue, The Galois Algebra with Galois Group which is the Automorphism Group, Journal of Algebra, 293(1) (2005), 312 - 318.

[5] G. Szeto and L. Xue, On Galois Algebras Satisfying the Fundamental Theorem, Communications in Algebra, to appear.

Received: November 29, 2006 